\title{
Terapia com betabloqueadores em pacientes após infarto do miocárdio e seus benefícios
}

\author{
Beta blockers therapy in patients after myocardial infarctions and its benefits \\ Terapia con betabloqueantes en pacientes después de um infarto de miocario y sus \\ beneficios
}

Brenda Isabelle Flamia ${ }^{1 *}$, Antony Rocha Porfírio ${ }^{2}$, Eduarda Pinheiro De Nadai ${ }^{3}$, Késia Martins da Cunha $^{3}$, Laís Rodrigues de Souza ${ }^{4}$, Leonardo Melhado Ziroldo ${ }^{5}$, Mateus Figueiredo Braga ${ }^{6}$, Nathany Dayrell Ferreira², Rícia Medeiros Palmeira de Araujo7, Valter Kuymjian ${ }^{8}$.

\section{RESUMO}

Objetivo: Analisar os aspectos da terapia com bloqueadores beta-adrenérgicos em pacientes após infarto do miocárdio e seus benefícios. Revisão bibliográfica: Os fármacos bloqueadores beta-adrenérgicos, chamados betabloqueadores, têm sido usados para tratar o infarto agudo do miocárdio por um decênio. São amplamente utilizados e demonstram resultados satisfatórios em termos de redução de mortalidade em pacientes após infarto agudo do miocárdio no período pré-reperfusão. No entanto, com o desenvolvimento e surgimento da terapia de intervenção percutânea primária (ICP), não há nenhum indicador que possa verificar a eficácia da duração do tratamento com betabloqueadores após infarto agudo do miocárdio (IAM). Considerações finais: $O$ uso de betabloqueadores após um IAM é uma conduta imprescindível e bem estabelecida, enquanto que a escolha por terapia de longa ou curta duração é foco de muitos estudos e permanece em controvérsias, apesar disso, demonstram redução da mortalidade após o infarto do miocárdio quando é feito o uso dos betabloqueadores em terapias curtas.

Palavras-chave: Antagonistas beta-adrenérgicos, Infarto do miocárdio, Duração da terapia.

\begin{abstract}
Objective: Analyze the aspects of beta-adrenergic blocker therapy in patients after myocardial infarction and its benefits. Bibliographic review: Beta-adrenergic blocking drugs, called beta-blockers, have been used to treat acute myocardial infarction for a decade. They are widely used and show satisfactory results reducing mortality in patients after acute myocardial infarction in the preperfusion period. However, with the development and emergence of primary percutaneous intervention therapy $(\mathrm{PCl})$, there is no indicator that can verify the efficacy of beta-blocker treatment duration after acute myocardial infarction (AMI). Final considerations: The use of betablockers after acute myocardial infarction is an essential and well-established approach, while the choice for long or short term therapy is the focus of many studies and remains controversial, despite that, demonstrate reduced mortality after myocardial infarction when the use of betablockers is done in short term therapies.
\end{abstract}

Keywords: Adrenergic beta-antagonists, Myocardial infarction, Duration of therapy.

\footnotetext{
1 Universidade do Planalto Catarinense (UNIPLAC), Lages - SC. *E-mail: brenda flamia@outlook.com

2 Universidade Federal dos Vales do Jequitinhonha e Mucuri (UFVJM), Diamantina - MG.

${ }^{3}$ Faculdade de Ensino Superior da Amazônia Reunida (FESAR), Redenção - PA.

${ }^{4}$ Centro Universitário UNIFAMINAS, Muriaé - MG.

${ }^{5}$ Faculdade Santa Marcelina (FASM), São Paulo - SP.

${ }^{6}$ Centro Universitário Maurício de Nassau (UNINASSAU), Recife - PE.

7 Universidade Federal do Rio Grande do Norte (UFRN), Caicó - RN.

8 Universidade Estadual de Londrina (UEL), Londrina - PR.
} 


\section{RESUMEN}

Objetivo: Analizar los aspectos de la terapia con bloqueadores beta-adrenérgicos en pacientes tras un infarto de miocardio y sus beneficios. Revisión bibliográfica: Los fármacos bloqueadores beta-adrenérgicos, llamados betabloqueantes, se utilizan desde hace una década para tratar el infarto agudo de miocardio. Se utilizan ampliamente y demuestran resultados satisfactorios en cuanto a la reducción de la mortalidad en pacientes tras un infarto agudo de miocardio en el periodo de preperfusión. Sin embargo, con el desarrollo y la aparición de la terapia de intervención percutánea primaria (ICP), no existe ningún indicador que pueda verificar la eficacia de la duración del tratamiento con betabloqueantes tras un infarto agudo de miocardio (IAM). Consideraciones finales: El uso de betabloqueantes después de un infarto agudo de miocardio es un enfoque esencial y bien estabelecido, mientra la elección de una terapia a largo o corto plazo es el centro de muchos estudios y sigue siendo controvertida, a pesar de esto, demuestran una reducción de la mortalidad después de un infarto de miocardio cuando el uso de betabloqueantes se hace en terapias cortas.

Palabras clave: Antagonistas adrenérgicos beta, Infarto del miocardio, Duración de la terapia.

\section{INTRODUÇÃO}

Os bloqueadores beta-adrenérgicos constituem uma das classes de drogas mais estudadas desde o seu surgimento, para diversas doenças cardiovasculares. Possuem evidência comprovada nas mais frequentes patologias cardiovasculares como a Hipertensão Arterial Sistêmica (HAS), a Insuficiência Cardíaca (IC) e na terapêutica da cardiopatia isquêmica (MARTíNEZ-MILLA J, et al., 2019). Seus efeitos antiarrítmicos também já foram comprovados fazendo parte do arsenal terapêutico das arritmias cardíacas, além de prevenir o remodelamento miocárdico (PARK JJ, et al., 2018).

Segundo Park JJ, et al. (2019), foram introduzidos na década de 1980, inicialmente para o tratamento de angina, e tiveram adoção em larga escala, sendo vistos como fundamentais no tratamento do infarto agudo do miocárdio (IAM). Durante a fase inicial de um IAM agudo, os bloqueadores beta-adrenérgicos agem reduzindo a extensão do infarto, estando associados a melhores desfechos cardiovasculares em curto prazo nos pacientes vítimas de IAM com supradesnivelamento do segmento ST (IAMCSST) (QUADROS AS, et al., 2016; ZEITOUNI M, et al., 2019). O primeiro fármaco da classe, o propranolol, foi sintetizado por Sir James Whyte Black em meados do século XX, inicialmente para o tratamento de angina, mas também passou a ser aplicado para o tratamento de arritmias, HAS e IAM (ZEITOUNI M, et al., 2019).

Em relação ao mecanismo farmacológico, compreende-se que os bloqueadores beta-adrenérgicos possuem a capacidade de antagonizar os $\beta 1$-adrenoceptores miocárdicos e renais, ou até mesmo ambos os adrenoceptores $\beta 1$ e $\beta 2$ localizados nos pulmões, na musculatura lisa e esquelética, levando ao bloqueio de estímulos adrenérgicos. Sendo assim, os principais benefícios desse antagonismo envolvem a redução da demanda de oxigênio e do trabalho miocárdico, consequentemente reduzem a angina, a extensão do infarto e o risco de óbito do paciente, além de possuir propriedades que reduzem as chances de fibrilação e taquicardia ventricular (PARK JJ, et al., 2018; ZEITOUNI M, et al., 2019).

Sabe-se que muitos avanços no tratamento do IAM aconteceram, sobretudo as intervenções coronárias percutâneas (ICPs), entretanto, os principais estudos que recomendam o uso de betabloqueadores, foram realizados antes do advento das ICPs, e na indisponibilidade da farmacoterapia adjuvante utilizada hoje, incluindo a dupla terapia antiplaquetária e os hipolipemiantes de alta potência, ressaltando a necessidade de pesquisas robustas nesse novo cenário (HARARI R e BANGALORE S, 2020).

De acordo com Zeitouni M, et al. (2019), registros norte-americanos e europeus, de pacientes que receberam alta com betabloqueadores após um IAM, mostram que cerca de $80 \%$ desses pacientes continuam fazendo uso da medicação em 1 ano, $70 \%$ em 2 anos e $58 \%$ em 3 anos. Esses dados heterogêneos evidenciam que as principais diretrizes não abordam de maneira clara e exata aspectos fundamentais a respeito dos bloqueadores beta-adrenérgicos, sobretudo a duração da terapêutica (OZASA N e KIMURA T, 2019).

Nesse contexto, estudos como os de Pedretti RFE (2018) colocam em questão a necessidade do uso desses medicamentos. Os principais motivos para tais questionamentos são a existência de terapias 
farmacológicas com maior eficácia como inibidores da enzima de conversão da angiotensina, antiplaquetários, anticoagulantes e reperfusão que quando comparadas, demonstram um efeito neutro dos betabloqueadores no prognóstico do paciente e prevenção de novo quadro.

A realização desse estudo justifica-se com o intuito de apresentar perspectivas atuais a respeito da terapia com bloqueadores beta-adrenérgicos no IAM, visto que os estudos antigos apresentam limitações importantes para a prática contemporânea. Com base no exposto, o presente estudo tem por objetivo analisar a terapia com bloqueadores beta adrenérgicos em pacientes após IAM e seus benefícios, através de uma revisão narrativa.

\section{REVISÃO BIBLIOGRÁFICA}

Apesar da evolução em tratamentos e intervenções surgidas com o passar dos anos, um dos principais motivos de óbito ao redor do mundo é o infarto agudo do miocárdio (IAM). O IAM frequentemente é causado por um bloqueio ou redução do fluxo sanguíneo a uma porção do coração, o que pode levar a necrose daquela região, ou seja, acontece a morte de miócitos em decorrência de uma isquemia miocárdica. Hoje já se reconhece que nem todas as causas de IAM necessariamente possuem uma etiologia vascular, podendo partir apenas da redução da oferta de oxigênio ao miocárdio (SALEH M e AMBROSE JA, 2018).

O principal mecanismo causador de IAM é a complicação de placa aterosclerótica, definido também como infarto tipo 1. Essa também é uma das principais causas de infarto em todo mundo (REED GW, et al., 2017). Ele pode ser dividido ainda em IAM com supradesnivelamento do segmento ST (IAMCSST) ou IAM sem supradesnivelamento do segmento ST (IAMSST), sendo que a existência do supra também continua sendo preditor de maior mortalidade (SALEH M e AMBROSE JA, 2018).

A taxa de mortalidade brasileira por essa patologia está entre uma das maiores do mundo, próxima a China e ao leste europeu (DOS SANTOS J, et al., 2018). Isso ainda acontece apesar da evolução quanto ao prognóstico e também do progresso que se obteve na melhoria de tratamentos (REED GW, et al., 2017). Muitos ainda têm sido os estudos randomizados acerca de terapias clínicas, porém estes, devido ao público incluído, numericamente, não são muito representativos (QUADROS AS, et al., 2016).

De acordo com Nambiar L e Meyer M (2018) os fármacos bloqueadores beta-adrenérgicos, denominados betabloqueadores, há décadas são utilizados como base para tratamentos do infarto agudo do miocárdio e doença arterial coronariana. Os resultados positivos do uso dessa classe foram evidenciados entre 1966 a 1991, previamente ao surgimento da intervenção percutânea. De acordo com o presente estudo, o uso de betabloqueadores propicia poucas vantagens se usados por pouco tempo, entre elas pode-se citar a melhora dos índices de mortalidade, porém, há grande taxa de reinfarto.

Esses medicamentos, propalados em 1960 como antianginosos, são usados como pilar para tratar diversas patologias do sistema cardiovascular, incluindo arritmias, insuficiência cardíaca e isquemias cardíacas. Ainda que a ação deles seja pautada no antagonismo de receptores beta-adrenérgicos, sabe-se que o efeito causado excede o mecanismo de ação (MARTíNEZ-MILLA J, et al., 2019).

Segundo Martínez-Milla J, et al. (2019) o uso de beta bloqueadores intravenosos em fase aguda do infarto agudo do miocárdio é benéfico, visto que há uma resposta do sistema nervoso simpático desencadeada pela dor, ansiedade e redução do débito cardíaco, levando a respostas negativas e risco de morte súbita. Entretanto, os benefícios dos betabloqueadores a longo prazo em pacientes com sequelas do infarto agudo do miocárdio, por exemplo, disfunção ventricular, ainda não estão bem evidenciados.

De acordo com Pedretti RFE (2018) estudos randomizados com 31 pacientes e controlados, evidenciaram diminuição em $23 \%$ na possibilidade de óbito, no caso de pessoas que estavam em uso de betabloqueadores. Dessa forma, diante do exposto é possível corroborar com diretrizes que preconizam o uso de betabloqueadores após IAM como método preventivo secundário, na ausência de contraindicações.

Os betabloqueadores foram muito utilizados, obtendo resultados satisfatórios no que tange aos índices de mortalidade em pacientes que sofreram previamente infarto agudo do miocárdio, no período pré-reperfusão. Entretanto, com a evolução e o advento da intervenção percutânea primária (ICP) não existem indicadores que validam a terapêutica prescrita de rotina com betabloqueadores após IAM (KIM J, et al., 2020). 
Além disso, conforme Hagsund T, et al. (2020), há poucos trabalhos que ressaltam os aspectos positivos acerca do uso dos betabloqueadores posterior ao moderno tratamento coronariano. A conduta mediante ao IAM foi marcadamente alterada ao decorrer dos anos, introduzindo terapias mais efetivas e maior uso de intervenções percutâneas.

Soeiro AM, et al. (2016) ressalta que as diretrizes estão de acordo ao uso de betabloqueadores caso haja reperfusão anterior ao IAM e uso de medicamentos terapêuticos atuais como os antiagregantes plaquetários e estatinas. O papel dessa classe de medicações na nova era tem sido questionado.

É possível notar que houve uma mudança na terapêutica para prevenção secundária ao ataque cardíaco e que se mostram mais eficientes, como o uso de inibidores da enzima conversora de angiotensina, estatinas, antiagregantes plaquetários e anticoagulantes. Somada à intervenção percutânea tanto em fase aguda do ataque cardíaco, como em fase crônica para casos de isquemia residual. Além disso, existe uma redução na adesão ao tratamento, visto que o paciente opta por quais fármacos tomar. Sendo assim, é questionável se os betabloqueadores são realmente indicados para todas pessoas com histórico prévio de IAM (PEDRETTI RFE, 2018).

Os ensaios BHAT E CAPRICORN, que utilizaram, respectivamente, carvedilol e propanolol, constataram haver redução da mortalidade em pessoas com insuficiência cardíaca (PEDRETTI RFE, 2018). Todavia, o ensaio COMMIT- 54 , além de ter mostrado que não há diminuição na mortalidade na administração precoce por um período curto de até 28 dias, mostrou não haver redução de mortalidade a longo prazo, apesar da redução de reinfarto e fibrilação ventricular (MARTíNEZ-MILLA J, et al., 2019; NAMBIAR L e MEYER M, 2018; PUYMIRAT E, et al., 2016).

Esse último ponto vai de encontro ao estudo retrospectivo realizado por Hagsund T, et al (2015), que apontou que pacientes que recebem alta sem um betabloqueador após um infarto do miocárdio têm uma taxa maior de readmissões para reinfarto após 1 ano do primeiro evento, quando comparados a pacientes que receberam a terapia medicamentosa na alta hospitalar.

Em pacientes submetidos a angioplastia primária, o estudo METOCARD - CNIC associou o uso de atenolol a uma menor extensão do infarto, bem como maior fração de ejeção a longo prazo, enquanto que o estudo EARLY-BAMI concluiu que não há efeitos cardioprotetores do atenolol (MARTíNEZ-MILLA J, et al., 2019). Essa discrepância de resultados está associada à diferença do momento em que o medicamento foi aplicado, sendo muito mais precoce no METOCARD- CNIC. Já o COMMIT-54 encontrou que o uso de intravenoso, de forma precoce, de beta bloqueador em IAM está associado a maior incidência de choque cardiogênico (MARTÍNEZ-MILLA J, et al., 2019; ZEITOUNI M, et al., 2019).

Nesse contexto, uma meta-análise de 2013 concluiu que o uso de betabloqueador intravenoso de forma precoce é seguro quando aplicado em pacientes sem sinais de insuficiência cardíaca (MARTíNEZ-MILLA J, et al., 2019). No entanto, isso contrasta com o estudo de Soeiro AM, et al. (2016), que encontrou diminuição na incidência do choque cardiogênico, além da redução da mortalidade intrahospitalar nas primeiras 24h após o episódio do IAM.

Todavia, em pacientes com fração de ejeção não reduzida ou sem insuficiência cardíaca, os efeitos de mortalidade dos betabloqueadores ainda não são bem estabelecidos (NAMBIAR L e MEYER M, 2018). Dois estudos de coorte já concluídos não encontraram benefícios do uso de betabloqueadores em pacientes sem insuficiência cardíaca ou disfunção ventricular esquerda, enquanto que os poucos estudos que existem atualmente - os ensaios clínicos REBOOT, BETAMI E REDUCE-SWEDEHEART- enfrentam limitações metodológicas e ainda não chegaram a uma conclusão (PUYMIRAT E, et al., 2016; PEDRETTI RFE, 2018).

Além desses estudos observacionais, uma meta-análise com 16.645 pacientes, realizada em 2016, também sugere que não há associação entre uso de betabloqueador em pacientes com fração preservada e diminuição da mortalidade (DONDO TB, et al., 2017). O que condiz com o resultado de uma meta-análise realizada em 2015, que chegou à conclusão de que os beta bloqueadores reduzem a mortalidade após infarto do miocárdio apenas em subgrupos específicos, dos quais se incluem aqueles com insuficiência cardíaca e fração de ejeção reduzida (PEDRETTI RFE, 2018; HAGSUND T, et al., 2020). 
Apesar disso, o registro FAST-MI (Registro Francês de Infarto Agudo do Miocárdio com Elevação de ST e sem Elevação de ST) chegou à conclusão de que, em pacientes sem insuficiência cardíaca, o uso precoce de betabloqueador foi associado a uma redução de mortalidade nos primeiros 30 dias, porém sem efeitos em aumento de mortalidade em casos de uso mais prolongado. Nessa perspectiva, um estudo de coorte publicado em 2017, que incluiu 180.000 pacientes da Inglaterra e do País de Gales, concluiu que o uso de betabloqueador após infarto em pacientes sem insuficiência cardíaca não reduz o risco de morte em nenhum momento após 1 ano da terapia (DONDO TB, et al., 2017).

Nesse sentido, é reconhecido que se deve ter cautela no uso de betabloqueadores, uma vez que eles podem reduzir a qualidade de vida do paciente em virtude dos seus principais efeitos adversos apontados pelas Diretrizes para Doença Cardíaca Isquêmica: fadiga, intolerância a exercícios físicos, insônia, pesadelos, letargia e impotência sexual (NAMBIAR L E MEYERM, 2018). Sendo assim, a Sociedade Europeia de Cardiologia recomenda o uso de betabloqueador após infarto agudo do miocárdio apenas em pacientes com disfunção ventricular esquerda (HAGSUND T, et al., 2020).

Na prática clínica da medicina há o questionamento quanto à duração do tratamento farmacológico com betabloqueadores em pacientes vítimas de IAM. Segundo Zeitouni M, et al. (2019) a dúvida em relação ao tempo de administração é decorrente da ausência de evidências científicas para os efeitos colaterais, os quais têm se apresentado recorrentemente em pacientes que são tratados com betabloqueadores à longo prazo, além da adesão ao tratamento. Também a mudança da recomendação posológica após a era da reperfusão miocárdica.

Vale a pena ressaltar que existem contraindicações específicas a este fármaco e, na vigência de tais condições eles não devem ser prescritos, sendo elas: frequência cardíaca menor que 60 batimentos por minuto, hipotensão, prolongamento do intervalo PR e antecedente de asma ou DPOC. Esse medicamento também deve ser evitado em caso de bloqueio atrioventricular de segundo ou terceiro grau, doença vascular periférica grave e disfunção ventricular grave (HAGSUND T, et al., 2020).

A divergência maior se dá para os eventos adversos que os betabloqueadores causam nos pacientes, conforme o estudo feito por Hwang D, et al. (2019), sendo eles: depressão, cefaleia, fadiga, hipotensão, bradicardia e doenças de vias aéreas. Por conta desses sintomas, hoje há prescrição de dosagens menores do medicamento, protegendo o paciente de novos eventos cardiovasculares além do auxílio em sua qualidade de vida.

O estudo feito por Sim HW, et al. (2020) mostrou que há redução de internação por insuficiência cardíaca e de mortalidade por consequências cardiovasculares com o uso de betabloqueador ao longo de 12 meses. Como conclui o estudo feito por Hwang D, et al. (2019), os benefícios da utilização do medicamento ao longo de 1 ano, porém levando em conta a dosagem, com doses baixas o paciente tem menores efeitos colaterais do fármaco além da taxa de que a sobrevida dos pacientes que receberam altas e baixas doses do fármaco são semelhantes, diferindo apenas dos pacientes que não fizeram uso.

É sabido que em pacientes que sofreram IAM, o uso do betabloqueador é recomendado, desde que ele não tenha nenhuma contraindicação aos efeitos do fármaco. A grande escala de prescrição do medicamento a seus pacientes é advinda de uma grande gama de estudos que mostram sua eficácia, oferecendo maior sobrevida a curto e longo prazo (PARK JJ, et al., 2018).

Como já supracitado, a duração ideal da terapia com betabloqueadores é desconhecida, contudo, além dos estudos que observaram seu grupo ao longo de 1 ano, Park JJ, et al. (2018) foi mais adiante e pesquisou as consequências do uso do fármaco ao longo de 3 anos pós IAM. O acompanhamento dos pacientes ao longo dos 3 anos rendeu muitos dados, entretanto, não conseguiu alcançar um resultado determinante quanto ao tempo de uso da droga. Seu estudo foi inconclusivo pois, o estudo mostrou resultados semelhantes em relação a sobrevida dos pacientes no intervalo de uso de 1 a 3 anos.

As doses de betabloqueador utilizadas na prática médica são significativamente mais baixas em comparação às utilizadas nos ensaios randomizados que determinam sua eficácia. Goldberger JJ, et al. (2015) realiza um estudo com um viés diferente dos anteriores. Sua pesquisa foca no questionamento de que 
se a dosagem do betabloqueador é diretamente proporcional com a sobrevida do paciente pós IAM. A diferenciação da dosagem para diferentes grupos de pacientes foi feita dividindo-os em 5 grupos, sendo: sem betabloqueador,> $0 \%$ a $12,5 \%,>12,5 \%$ a $25 \%,>25 \%$ a $50 \%$ e> $50 \%$ da dose alvo.

Os resultados foram que as terapias de doses baixas, que são as $\leq 25 \%$, têm significativamente menor taxa de mortalidade em comparação com as terapias de dosagem alta, $\geq 50 \%$. Doses mais altas como as usadas nos ensaios determinantes para a dosagem do medicamento não demonstram aumento de sobrevida do paciente em comparação às doses mais baixas, as quais são mais utilizadas na prática médica. O resultado deste estudo demonstra o quanto ainda deve-se estudar a dosagem dos betabloqueadores para tratamento pós IAM (GOLDBERGER JJ, et al., 2015).

Comparações entre a duração curta ou longa do uso de betabloqueadores é sempre foco de estudos e permanece sendo ainda controverso. Betabloqueadores frequentemente são prescritos na prática clínica com duração superior a um ano para os pacientes sem contraindicações, mas, o mais difícil, sem dúvidas, é saber quando suspender o uso.

Em estudo, Kim J, et al. (2020), demonstrou que o uso de betabloqueadores por mais de um ano resultou em menor risco de morte por todas as causas quando comparado ao uso menor que um ano. Corrobora com essa afirmativa estudos realizados por Hagsund T, et al. (2020) e Park JJ, et al. (2018) que afirmam que os efeitos benéficos em terapias curtas são bem estabelecidos, reforçando a redução da mortalidade.

Contudo, baseando-se em Hagsund T, et al. (2020) pacientes em acompanhamento por um ano não apresentam diferença analisando-se a insuficiência cardíaca quando comparado a pacientes que não utilizaram betabloqueadores. Sabe-se ainda que os pacientes que não utilizam essa medicação possuem taxas de readmissão hospitalares maiores no ano após o evento e também maior mortalidade.

As terapias a longo prazo, 3 anos ou mais de uso, não demonstram benefícios em estudos atuais para pacientes não complicados (KIM J, et al., 2020; PARK JJ, et al., 2018). Alguns autores apontam que apenas pacientes com fração de ejeção reduzida, insuficiência cardíaca e disfunção sistólica do ventrículo direito podem ser os únicos beneficiados com melhora da sobrevida em terapias longas (PARK JJ, et al., 2018).

Para mais, embasando-se em Zeitouni M, et al. (2019) é possível afirmar que os grandes estudos longitudinais modernos não fornecem evidências suficientes que corroborem com a terapia a longo prazo em pacientes não complicados. Portanto, reitera-se que faltam evidências robustas sobre benefício clínico da terapia longa após reperfusão miocárdica bem-sucedida e com função cardíaca normal.

As limitações dos estudos já realizados sobre essa temática dizem respeito principalmente ao diferente perfil de risco dos pacientes incluídos em grupos nos grupos de estudos (KIM J, et al., 2020; PARK JJ, et al., 2018). Além de alguns não mensurar outros fatores de risco cardiovascular como história familiar, prática de atividade física, doenças inflamatórias autoimunes (HAGSUND T, et al., 2020).

Ko DT e Jackevicius CA (2018) pontuam ainda que a duração ótima do tratamento deverá ser uma decisão médica adaptada a cada paciente em particular, analisando suas especificidades. Fato é que o uso de betabloqueadores após infarto do miocárdio é recomendado (nível de evidência $\mathrm{B}$, classe II), conforme abordado nesta revisão, com exceção de pacientes com contraindicações ao uso, possuindo benefícios bem esclarecidos por diversos estudos (HAGSUND T, et al., 2020; PARK JJ, et al., 2019).

\section{CONSIDERAÇÕES FINAIS}

As principais diretrizes não abordam de maneira clara e precisa aspectos a respeito dos betabloqueadores, sobretudo a duração e terapêutica, uma vez que a conduta médica do IAM foi notoriamente alterada ao longo dos anos com introdução das terapias mais efetivas e com um maior uso de intervenções percutâneas. $\mathrm{Na}$ prática clínica, betabloqueadores são repetidamente prescritos, com duração do tratamento superior a um ano em pacientes sem contraindicações. A escolha por terapia de longa ou curta duração é foco de muitos estudos e permanece em controvérsias, estudos demonstram menor risco de morte após o IAM quando o uso é feito por menos que um ano, ratificando essa afirmação, estudos afirmam efeitos benéficos em terapias curtas bem estabelecidas, ressaltando a redução da mortalidade. 


\section{REFERÊNCIAS}

1. DONDO TB, et al. $\beta$-Blockers and Mortality After Acute Myocardial Infarction in Patients Without Heart Failure or Ventricular Dysfunction, 2017; 69(22): 2710-2720.

2. DOS SANTOS J, et al. Mortalidade por infarto agudo do miocárdio no Brasil e suas regiões geográficas: análise do efeito da idade-período-coorte, 2018; 23(5): 1621-1634.

3. GOLDBERGER JJ, et al. Effect of Beta-Blocker Dose on Survival After Acute Myocardial Infarction, 2015; 66(13): 14311441.

4. HAGSUND T, et al. $\beta$-blockers after myocardial infarction and 1-year clinical outcome - A retrospective study, 2020; 20(1): 165.

5. HARARI R, BANGALORE S. Beta-blockers after acute myocardial infarction: an old drug in urgent need of new evidence! 2020.

6. HWANG D, et al. Prognostic Impact of $\beta$-Blocker Dose After Acute Myocardial Infarction, 2019; 25;83(2): 410-417.

7. KIM J, et al. Long-Term $\beta$-blocker therapy and clinical outcomes after acute myocardial infarction in patients without heart failure: Nationwide cohort study, 2020; 41(37): 3521-3529.

8. KO DT, JACKEVICIUS CA. Stopping $\beta$-blockers after myocardial infarction, 2018; 11(4): e004678.

9. MARTÍNEZ-MILLA J, et al. Papel de los bloqueadores beta en la enfermedad cardiovascular en 2019, 2019.

10. NAMBIAR L, MEYER M. $\beta$-Blockers in myocardial infarction and coronary artery disease with a preserved ejection fraction: recommendations, mechanisms, and concerns. Coron Artery Dis, 2018; 29(3): 262-270.

11. OZASA N, KIMURA T. Oral B-Blocker Therapy in Acute Myocardial Infarction, 2019; 83: 281-282.

12. PARK JJ, et al. Effect of B-Blockers Beyond 3 Years After Acute Myocardial Infarction, 2018; 7(5): e007567.

13. PARK JJ, et al. Differential Effect of beta-blockers According to Heart Rate in Acute Myocardial Infarction Without Heart Failure or Left Ventricular Systolic Dysfunction: A Cohort Study, 2019; 94(12): 2476-2487.

14. PEDRETTI RFE. Beta-blockers after myocardial infarction: Are they useful to all patients? And how long should be the beta-blocker therapy? 2018; 88:971.

15. PUYMIRAT E, et al. $\beta$ blockers and mortality after myocardial infarction in patients without heart failure: multicentre prospective cohort study, 2016; 354: 480 .

16. QUADROS AS, et al. Infarto Agudo do Miocárdio na Prática Clínica Diária, 2016; 29(4):253-26.

17. REED GW, et al. Acute myocardial infarction, 2017; 389(10065): 197-210.

18. SALEH M, AMBROSE JA. Understanding myocardial infarction [version 1; referees: 2 approved], $2018 ; 1378$.

19. SIM HW, et al. Beta-blockers and renin-angiotensin system inhibitors in acute myocardial infarction managed with inhospital coronary revascularization, 2020; 10:15184.

20. SOEIRO AM, et al. Mortality reduction with use of oral beta-blockers in patients with acute coronary syndrome, 2016; 71(11): 635-638.

21. ZEITOUNI M, et al. Do Patients need Lifelong $\beta$-Blockers after an Uncomplicated Myocardial Infarction? 2019; 19(5): 431-438. 